\title{
Coupled aggregation of mitochondrial single-strand DNA-binding protein tagged with Eos fluorescent protein visualizes synchronized activity of mitochondrial nucleoids
}

\author{
TOMÁŠ OLEJÁR, DAVID PAJUELO-REGUERA, LUKÁŠ ALÁN, ANDREA DLASKOVÁ and PETR JEŽEK \\ Department of Membrane Transport Biophysics, Institute of Physiology, \\ The Czech Academy of Sciences, Prague 14220, Czech Republic
}

Received October 20, 2014; Accepted June 5, 2015

DOI: $10.3892 / \mathrm{mmr} .2015 .4085$

\begin{abstract}
Oligomer aggregation of green-to-red photoconvertible fluorescent protein Eos (EosFP) is a natural feature of the wild-type variant. The aim of the present study was to follow up mitochondrial nucleoid behavior under natural conditions of living cells transfected with mitochondrial single-strand DNA-binding protein (mtSSB) conjugated with EosFP. HEPG2 and SH-SY5Y cells were subjected to lentiviral transfection and subsequently immunostained with anti-DNA, anti-transcription factor A, mitochondrial (TFAM) or anti-translocase of the inner membrane 23 antibodies. Fluorescent microscopy, conventional confocal microscopy, superresolution biplane fluorescence photo-activation localization microscopy and direct stochastic optical reconstruction microscopy were used for imaging. In the two cell types, apparent couples of equally-sized mtSSB-EosFP-visualized dots were observed. During the time course of the ongoing transfection procedure, however, a small limited number of large aggregates of mtSSB-EosFP-tagged protein started to form in the cells, which exhibited a great co-localization with the noted coupled positions. Antibody staining and 3D immunocytochemistry confirmed that nucleoid components such as TFAM and DNA were co-localized with these aggregates. Furthermore, the observed reduction of the mtDNA copy number in mtSSB-EosFP-transfected cells suggested a possible impairment of nucleoid function. In conclusion, the present study demonstrated that coupled nucleoids are synchronized by mtSSB-EosFP overexpression and visualized through their equal binding capacity to mtSSB-EosFP-tagged protein. This observation suggested parallel replication and transcription activity of nucleoid couples native from a parental one.
\end{abstract}

Correspondence to: Dr Tomáš Olejár, Department of Membrane Transport Biophysics, No. 75, Institute of Physiology, The Czech Academy of Sciences, Vídenská 1083, Prague 14220, Czech Republic E-mail: tomas.olejar@seznam.cz

Key words: mitochondrial nucleoid, single-stranded DNA-binding protein, photoconvertible fluorescent protein Eos
Preserved coupling in late stages of artificial EosFP-mediated aggregation of tagged proteins suggested a rational manner of mitochondrial branching that may be cell-type specifically dependent on hierarchical nucleoid replication.

\section{Introduction}

Mitochondrial (mt) nucleoids as functional units are composed of circular DNA coding 13 genes involved in the electron transport chain. The DNA is maintained by several nuclear-encoded proteins. Topically, it is attached to the inner mitochondrial membrane (1). The basic nucleoid-composing proteins are responsible for transcription, replication and repair. A detailed overview of their function, composition and structure, which exceeds the capacities of the present study, was given by Bogenhagen (2). Mitochondrial transcription factor A (TFAM) is responsible for mtDNA binding and space conformation (3), stabilization of the mitochondrial genome (4) and transcription (5). TFAM regulates the mt genome copy number (6). In certain cases, its overexpression rescues this copy number and restores the activity of respiratory chain-associated molecules and ATP synthesis, resulting in restoration of insulin secretion in Pdx1-defective INS1 cells (7) or protection of 3T3-L1 adipocytes from NYGGF4 (PID1) overexpression-induced insulin resistance (8). mtSSB protein is expressed in the nucleoids of mammalian mitochondria (9) and is responsible for replication and repair of mtDNA and also for mtDNA maintenance (10). It acts via covering single-stranded mtDNA (11), and by enhancing mtDNA polymerase (12) and helicase (13) activity. Twinkle is an mtDNA helicase which is, in a complex with mtDNA polymerase and mtSSB, responsible for unwinding mtDNA (14). Transcription and replication is then performed by specific mitochondrial RNA or DNA polymerases $(15,16)$. Distribution and nucleoid interrelation during physiological and pathophysiological conditions of fission/fusion processes have also been studied (17). Disturbance in nucleoid components and mutations in mtDNA were identified to be significant in various diseases $(18,19)$, including carcinogenesis $(20)$.

Photoactivable proteins, including green, yellow and cyan fluorescence protein as well as Keima tagged to nucleoid protein components are widely used for studying these mtDNA-containing units (21). The properties of green-to-red 
photoconvertible fluorescent protein Eos (EosFP) are utilized in a novel method of superresolution fluorescence photo-activation localization microscopy (fPALM) (22). However, oligomer aggregation is a natural effect of the wild-type (WT) protein variant (23). The aim of the present study was to follow up mitochondrial nucleoid behavior under natural conditions of living cells transfected with mtSSB wild-type EosFP-tagged protein, also with regard to the contents of other nucleoid components (mtDNA, TFAM).

\section{Materials and methods}

DNA vectors and lentiviral (LTV) particle production. The ORF of mtSSB was purchased from Invitrogen Life Technologies (Carlsbad, CA 92008, USA), amplified by polymerase chain reaction (PCR) with attb primers and sub-cloned into modified pLenti 6.3/V5-DEST (Invitrogen Life Technologies) vector with dimeric EosFP enabling N-terminal fusion after LR recombination. Lentiviral particles based on mtSSB-EosFP plasmids were multiplied in the 239LTV cell line using common calcium phosphate transfection utilizing the packaging plasmids LP1, LP2 and VSV-G (Invitrogen Life Technologies). The lentiviral stock was filtered and concentrated by PEG-it Virus Precipitation Solution (System Biosciences, Mountain View, CA, USA).

Cell cultures. The human hepatocellular cancer HEPG2 cell line (European Collection of Cell Cultures, Salisbury, UK; 85011430) was maintained as described previously (17). The human neuroblastoma SH-SY5Y cell line (American Type Culture Collection, Manassas, VA, USA; CRL-2266) was cultivated at $37^{\circ} \mathrm{C}$ in humidified air with $5 \% \mathrm{CO}_{2}$ in glucose-free DMEM medium (Invitrogen Life Technologies) supplemented with $2 \mathrm{mM}$ glutamine (Sigma-Aldrich, St. Louis, MO, USA), $0.5 \mathrm{mM}$ sodium pyruvate (Sigma-Aldrich), $15 \%$ $(\mathrm{v} / \mathrm{v})$ fetal calf serum (Biochrom GmbH, Berlin, Germany), $10 \mathrm{mM}$ 4-(2-hydroxyethyl)-1-piperazineethanesulfonic acid (Sigma-Aldrich), $100 \mathrm{IU} / \mathrm{ml}$ penicillin (Sigma-Aldrich), $100 \mu \mathrm{g} / \mathrm{ml}$ streptomycin (Sigma-Aldrich) and $11 \mathrm{mM}$ glucose (Sigma-Aldrich). For microscopy, HEPG2 and SH-SY5Y cells were cultured for 3-5 days or 2-4 days, respectively, on glass coverslips coated with poly-L-lysine.

Primary polyclonal antibodies. Rabbit anti-human anti-TFAM antibody was kindly provided by Professor D.F. Bogenhagen (Department of Pharmacological Sciences, University at Stony Brook, Stony Brook, NY, USA). Rabbit anti-human anti-mtSSB was purchased from Sigma-Aldrich (cat. no. HPA002866), mouse anti-human anti-DNA was obtained from Progen Biotechnik GmbH (Heidelberg, Germany; cat. no. 61014) and mouse anti-human anti-TIM 23 was from BD Biosciences (Franklin Lakes, NJ, USA; cat. no. 611222).

Immunocytochemistry and confocal microscopy. Samples were fixed with cooled $\left(4^{\circ} \mathrm{C}\right) 4 \%$ paraformaldehyde in phosphate-buffered saline (PBS) overnight and washed three times for $10 \mathrm{~min}$ with PBS. The samples were then permeabilized for $1 \mathrm{~h}$ with PBS $+0.1 \%$ (w/v) Triton X-100 0.1\% (w/v) Tween 20 and $0.3 \mathrm{M}$ glycine (all from Sigma-Aldrich). Coverslips were blocked with 5\% bovine serum (Sigma-Aldrich) for $1 \mathrm{~h}$ and incubated

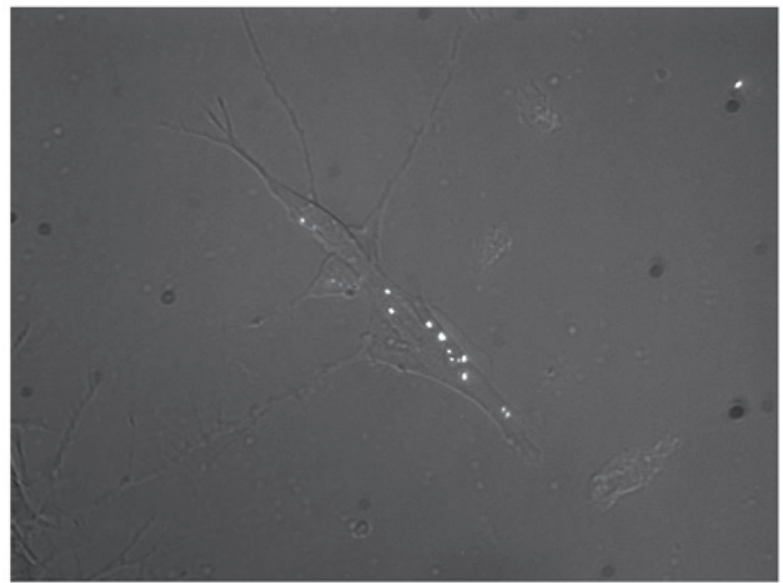

Figure 1. The first mitochondrial single-strand DNA-binding protein/Eos fluorescent protein nucleoids (white) appearing on day 2 after lentiviral transduction in SHSY5Y cells. Clearly visible coupling of equally-sized nucleoids in the cytoplasm of individual cells suggests the same parallel binding capacity of neighboring nucleoids. Fluorescent microscopy; original magnification, $\mathrm{x} 400$.

overnight at $4{ }^{\circ} \mathrm{C}$ with the appropriate primary antibody. Immunostaining with primary anti-TFAM or anti-DNA was followed with secondary Alexa-647- or Alexa-568-conjugated antibodies (Invitrogen Life Technologies).

EosFP was excited at $488 \mathrm{~nm}$ and Alexa-568-conjugated antibody was excited at $543 \mathrm{~nm}$ using an Olympus IX81 fluorescent microscope (Olympus, Tokyo, Japan). An inverted confocal fluorescent Leica TCS SP2 AOBS microscope (Leica Microsystems Inc., Wetzlar, Germany) was used with a PL APO 100x/1.40-0.70 oil immersion objective (a pinhole of 1 Airy unit). EosFP was excited at $488 \mathrm{~nm}$ with a $20-\mathrm{mW}$ argon laser, whereas Alexa-647-conjugated antibodies were excited at $630 \mathrm{~nm}$ with a $1.2-\mathrm{mW}$ HeNe laser.

Biplane fPALM and direct stochastic optical reconstruction microscopy (dSTORM) superresolution microscopy. Samples were visualized with a Biplane PPALM instrument, a prototype of Vutara (SR-200; Vutara Inc., Salt Lake City, UT, USA) with settings described previously (24). The composition of the dSTORM imaging buffer was $10 \%$ glucose, 169 units glucose oxidase, 1.4 units catalase and $50 \mathrm{mM}$ Tris- $\mathrm{HCl}(\mathrm{pH} 8.0)$ (all from Sigma-Aldrich), all in $10 \mathrm{mM} \mathrm{NaCl}$ (Lach-Ner, Neratovice, Czech Republic).

$m t D N A$ copy number. The number of mtDNA copies per cell was determined by qPCR as described previously (25) with human primer sequences as follows: Nuclear forward, 5'GGCAGCTTTGAAGAACGGGAC3' and reverse, 5'CACAGGGTTAGGAGGCAGCAA3'; mitochondrial forward, 5'CAGTCTGCGCCCTTACACAAAA3' and reverse, 5'TGGACCCGGAGCACATAAATA3'. The SYBR Green (Promega Corp., Madison, WI, USA) PCR detection was performed on a LightCycler ${ }^{\circledR} 480$ Instrument, with LightCycler software version 1.5 (Roche Diagnostics Ltd., Basel, Switzerland).

Western blot analysis. Immunoblot analyses were performed using respective cell lysates; proteins were separated by $12 \%$ SDS-PAGE and transferred onto a polyvinylidene difluoride 
A

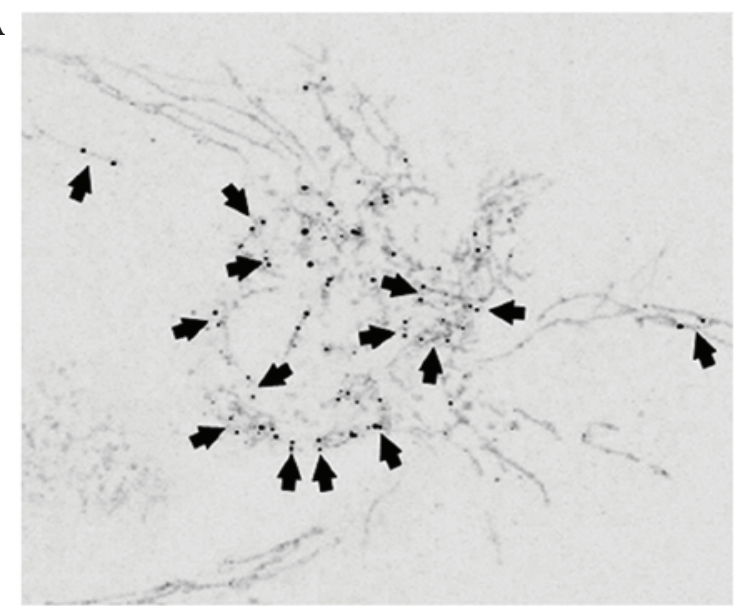

B

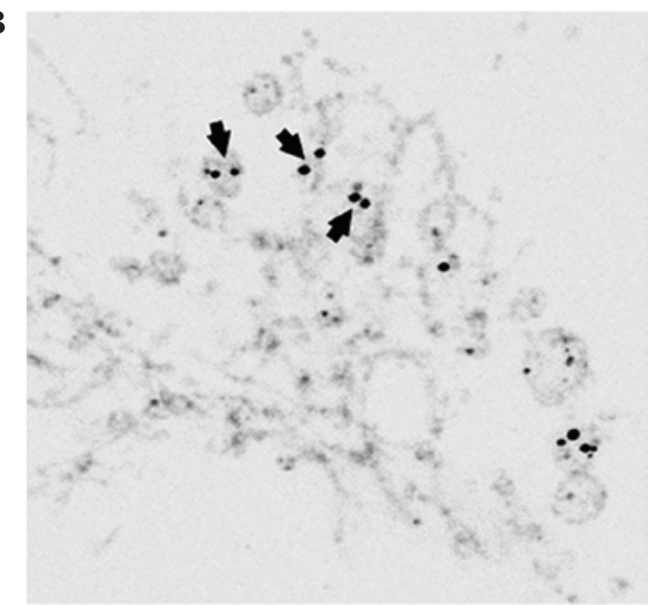

Figure 2. Later mtSSB-EosFP nucleoids (black dots) in HEP G2 cells after lentiviral transduction, counterstained with anti TIM23 antibody (grey fibers). Depending on mitochondrial activity in individual cells and time course, coupled nucleoid figures (see arrows) can be with or without marked aggregation, but a certain level of coupling is protected even in later stages. mtSSB-EosFP nucleoid couples can be seen either (A) in tubular mitochondria on day 4 , but also (B) in mitochondrial bulbs on day 5. Confocal microscopy; original magnification, x1,000. mtSSB-EosFP, mitochondrial single-strand DNA-binding protein/Eos fluorescent protein; TIM, translocase of the inner membrane.

membrane (Bio-Rad Laboratories, Hercules, CA, USA). The total quantity of protein was measured using a Bicinchoninic Acid Assay kit (Sigma-Aldrich) and $30 \mu \mathrm{g}$ total protein was loaded into each lane. Blots were blocked with $5 \%$ bovine serum, incubated with a primary antibody overnight at $4^{\circ} \mathrm{C}$, washed again, and the membrane was incubated with horseradish peroxidase-conjugated secondary antibody for $1 \mathrm{~h}$ at room temperature. Proteins were visualized using Amersham Enhanced Chemiluminescence Western Blotting Detection Reagent (GE Healthcare Life Sciences, Little Chalfont, UK) and a LAS-1000 (Fujifilm Life Science, Tokyo, Japan).

Statistical analysis. T-tests were conducted using SigmaPlot software, version 9 (Systat Software, Inc., San Jose, CA, USA). $\mathrm{P}<0.05$ was considered to indicate a statistically significant difference.

\section{Results}

Transcription efficacy and construct confirmation. Between the two cell types, a difference in the time course of mtSSB-EosFP appearance was noted: While in SH-SY5Y cells, the first fluorescent dots were already observed on day 1 (within the first 48 h; Fig. 1), the first fluorescent dots in HEPG2 cells were visible late on day 3 after lentiviral transduction. Furthermore, a difference in lentiviral transduction efficacy was observed between the two cell lines: In SH-SY5Y, $40-50 \%$ of cells were fluorescent on day 4, while in HEPG2 cells, only 5-10\% were fluorescent on day 4 and/or day 5 . Western blot analysis confirmed an additional mtSSB-positive band corresponding to the molecular weight of this protein $(17 \mathrm{kDa})$ plus $26 \mathrm{kDa}$ of EosFP (data not shown). The results did not show any significant difference in the natural mtSSB content between transduced and control samples of the two cell lines.

\section{Microscopic observations}

Early $m$ tSSB-EOSFP nucleoids in SH-SY5Y and HEPG2 cells. Depending on the time course of lentiviral transduction in the individual cell line, the first coupled mtSSB-EosFP dots were observed in the two cell lines on day 1 and day 3, respectively, following transduction (day 0). The observation of mtSSB-EosFP nucleoid coupling was confirmed by common fluorescent microscopy and fPALM superresolution microscopy.

Later mtSSB-EosFP nucleoids and immunocytochemistry in SH-SY5Y and HEPG2 cells. Generally, coupling in later stages following lentiviral treansduction was recorded at high rates, but not in absolutely all nucleoid figures in $100 \%$ of all cells; however, equal twins of variable size were clearly distinguished.

TIM23. As verified by confocal and superresolution microscopy approaches, mtSSB-Eos-coupled nucleoids (late as well as early) were located in the mitochondria as confirmed by the anti-TIM23 antibody (Figs. 2 and 3A). In later stages (days 4 and 5) in HEPG2 cells, the size of nucleoid couples differed among cells and, depending on mitochondrial morphology, were located either in tubular mitochondria or mitochondrial bulbs (Fig. 2).

$m t D N A$. Using confocal microscopy, co-localization of mtSSB-EosFP nucleoid couples with mtDNA was proven. In the two cell types, co-localization of mtSSB-EosFP together with mtDNA was observed. In SH-SY5Y cells on day 4, almost all mtDNA was attached to a limited number of accumulated and enlarged mtSSB-EosFP aggregates close to the nucleus (Fig. 3B) in which, however, coupling was preserved as described above. However, in HEPG 2 cells on day 4 and/or day 5, the presence of accumulations of copied mtDNA coupled to accumulations of mtSSB-EosFP without topical predilection; however, not all mtDNA was attached and a dominant proportion of common wild-type mtDNA nucleoids remained unaffected (Fig. 3B). However, accumulations of mtDNA co-localization with mtSSB-EosFP were also enlarged. In compliance with TIM23 co-localization, mtSSB-EosFP couples were observed to either be coupled in mtDNA chains, suggesting tubular mitochondria, but also in mtDNA clusters, suggesting the presence of mitochondrial bulbs. 
A

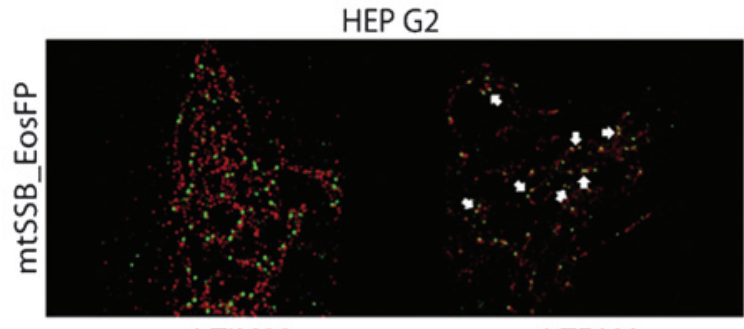

anti-TIM23

B SH-SY5Y HEP G2

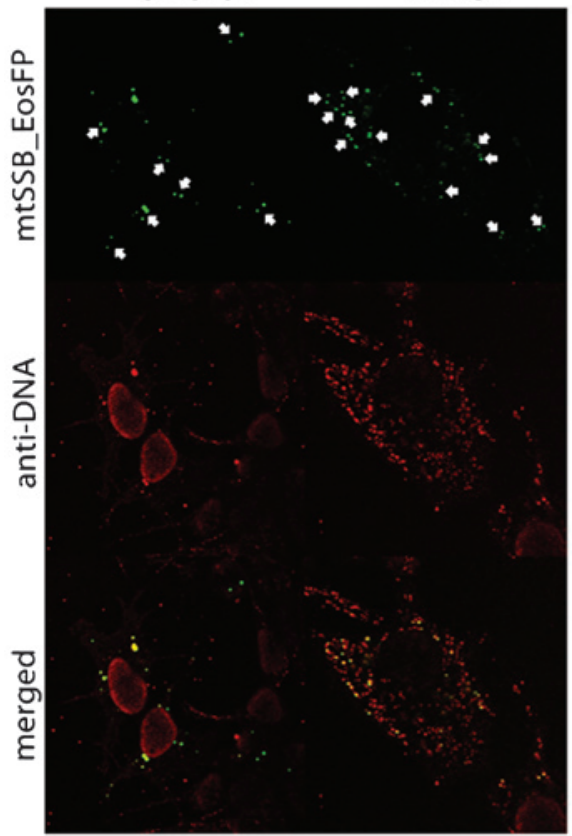

anti-TFAM

C

SH-SY5Y

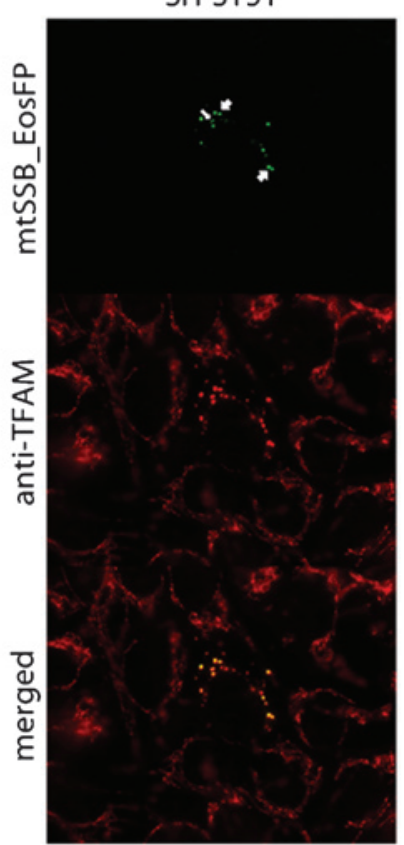

Figure 3. (A) Superresolution microscopy visualizing early coupled mtSSB-EosFP nucleoids (green) with TIM23 counterstain (red) in HEP G2 cells and coupled mtSSB-EosFP nucleoids (green) surrounded by TFAM (red) in HEP G2 cells on day 3 after lentiviral transduction. Clerarly visible are couples in mitochondria couterstained with antibody against TIM23. The coupling counterstained with TFAM is indicated by arrows. (B) mtSSB-EosFP nucleoids (green) in SH-SY5Y cells on day 4 after lentiviral transduction. Counterstaining with antibody against DNA (red) proved co-localization of mtSSB-EosFP protein construct with mtDNA. Eos-mediated aggregation of mtSSB is accompanied by the aggregation of mtDNA in transducted cells. This mtDNA aggregation is not observed in cells without the presence of EosFP. However, couples without counterstaining with mtDNA are also observed. Preservation of coupling (arrows) in later stages of aggregation together with the presence of larger mtSSB-EosFP-coupled masses in central parts of cells also suggests certain levels of hierarchy in nucleoid organization. Confocal microscopy; original magnification, x400. However, in HEPG2 cells, different mtSSB-EosFP nucleoids (green) were observed after lentiviral transduction. Counterstaining with antibody against DNA (red) proved co-localization of mtSSB-EosFP protein construct with mtDNA. Accumulations of mtDNA were present together with coupled accumulations of mtSSB-EosFP without topical pre-dilection; however, in these cells, not all mtDNA was attracted to mtSSB-EosFP and a dominant proportion of common wild-type mtDNA nucleoids remained unaffected. However, the mass of mtDNA co-expressed with mtSSB-EosFP was also increased. Similarly to SH-SY5Y, mtSSB-EosFP-mediated coupling (arrows) was preserved until later stages on day 5. Confocal microscopy; original magnification, x1,000. (C) mtSSB-EosFP-coupled nucleoid aggregation (green) in SH-SY5Y cells on Day 4 after lentiviral transduction (arrows). Counterstaining with antibody against human TFAM (red) proved co-localization of mtSSB-EosFP protein construct with TFAM. Similarly to the observation with anti-DNA counterstaining, Eos-mediated aggregation of mtSSB was accompanied by the aggregation of TFAM in transducted cells. This TFAM aggregation was not observed in the other cells without the presence of EosFP, and the preservation of coupling was also recorded. mtSSB-EosFP, mitochondrial single-strand DNA-binding protein/Eos fluorescent protein; TIM, translocase of the inner membrane; TFAM, transcription factor A, mitochondrial.

TFAM. mtSSB-EosFP and TFAM co-localization was observed since the early stages of lentiviral transduction in the two cell lines (Fig. 3A). In later stages of lentiviral transduction, aggregation of mtSSB-EosFP and TFAM, similar to that of mtDNA accumulations, was observed mostly in the SH-SY5Y cell line (Fig. 3C). Cells without the presence of mtSSB-EosFP did not show any TFAM aggregation.

mtDNA copy number. The mtDNA copy number per cell decreased from $100 \%$ [standard deviation $(\mathrm{SD})=6.44 \%$ ] in the control SH-SY5Y cells to $79.63 \%$ (SD=7.79\%) with statistically significant difference between the groups $(\mathrm{P}=0.007)$. In HEPG2 cells, the level decreased from $100 \%$ ( $\mathrm{SD}=2.80 \%$ ) to $82.06 \%$ $(\mathrm{SD}=4.48 \%)$ with statistically significant difference between the groups $(\mathrm{P}=0.007)$.

\section{Discussion}

The present study reported the cell-by-cell observation of a relatively high rate of nucleoid coupling and subsequent aggregation mediated by mtSSB tagged with wild-type EosFP. This effect was accompanied by aggregation of mtDNA and TFAM, suggesting an important role of mtSSB in nucleoid/mtDNA distribution within the mitochondrial network. These aggre- 
gates are fully located to the mitochondria, co-localizing with TIM23 protein.

It is also known that mtSSB itself influences mitochondrial biogenesis (26). Overexpression of this nucleoid component caused the re-distribution of the mitochondria to a perinuclear location, implying its specialized function in cellular metabolism. mtSSB overexpression also led to increased mitochondrial fragmentation (27). However, co-localization of mtDNA with dynamin-related protein (DRP)1, the membrane component responsible for mitochondrial fission, in the context of the immediate transmembrane neighborhood of mtDNA and cytoplasmatic cytosceletal components, including tubulin and/or the associated transporter kinesin (28), suggests clear evidence of nucleoid-organized mitochondrial biogenesis and branching. DRP1 knockdown caused nucleoid clustering and aggregation in HeLa cells, suggesting prevention of this effect and possible maintenance of mtDNA quality by mitochondrial fission (29). According to the observations of the present study, aggregation of mtSSB-EosFP nucleoids governing the distribution of either mtDNA or TFAM in parallel suggests the possible leading role of natural mtSSB in mtDNA/nucleoid distribution within the mitochondrial network. Aggregation of mtSSB-EosFP with TFAM or mtDNA in a couple suggested a key role of $\mathrm{mtSSB}$ as an organizational core for nucleoid location within the network, in parallel utilizing tetramerization as a natural effect of mtSSB together with the ability of its C-terminal to interact with other proteins $(30,31)$. However, a decrease of the mtDNA copy number in SH-SY5Y and HEPG2 cells suggested improper nucleoid replication in mtSSB-EosFP lentivirus-transduced cells.

In spite of this, mtSSB-EosFP overexpression visualized clear coupling of nucleoids in HEPG2 and SH-SY5Y cells. Nucleoid couples observed in the present study most probably arose from equal mtSSB binding capacity of neighboring nucleoids, resulting either from equal mtDNA content and/or transcription/replication activity and may indicate an important harmonization of their activity. Preservation of this nucleoid coupling in stages of aggregates, as described above, suggested a certain topical affiliation, and together with the observation in SH-SY5Y cells that larger couples are located perinuclearly, also a certain level of hierarchical organization. In HEPG2 cells, mtSSB-EosFP aggregation and coupling was observed without topic perinuclear predilection, but was randomly dispersed within the mitochondrial network. This organization is, however, dependent on the mode of mitochondrial branching, mitochondrial activity/metabolism and possibly also on the different levels of mitophagy in individual cell types. It has already been proven that Twinkle-dependent mtSSB co-localizes with only a subset of nucleoids (32). In the present study, this observation was confirmed for HEPG2, but not in SH-SY5Y cells, where the two investigated nucleoid components (mtDNA and TFAM) were fully attracted to mtSSB-EosFP aggreagates. An interesting observation was made in HEPG2 cell, where couples were identified either in nucleoids in tubular mitochondria, but also in nucleoid clusters located in mitochondrial bulbs that are even physiologically present in this cell type.

The differential susceptibility to lentiviral transduction between SH-SY5Y and HEPG2 cells cannot be easily and precisely explained; however, their differential mitochondrial metabolism, mtDNA replication and/or transcription activity, number, composition and behavior of natural mtDNA nucloids, mitophagy and numerous others must be taken into account.

The present study pointed out possible essential mechanisms of synchronized activity of nucleoids visualized by artificial mtSSB-EosFP coupling. Subsequent to mtSSB-EosFP-mediated aggregation of these neighboring mtDNA-organizing units containing other components, including mtDNA and TFAM, suggests a leading role of mtSSBs in their distribution within the mitochondrial network.

\section{Acknowledgements}

This work was supported by The Centre of Biomedical Research (CZ.1.07/2.3.00/30.0025) and by the Grant Agency of the Czech Republic (grant nos. 13-02033 and P302/10/0346). This project was co-funded by the European Social Fund and the state budget of the Czech Republic.

\section{References}

1. Brown TA, Tkachuk AN, Shtengel G, Kopek BG, Bogenhagen DF, Hess HF and Clayton DA: Superresolution fluorescence imaging of mitochondrial nucleoids reveals thein spatial range, limits and membrane interaction. Mol Cell Biol 31: 4994-5010, 2011.

2. Bogenhagen DF: Mitochondrial DNA nucleoid structure. Biochim Biophys Acta 1819: 914-920, 2012.

3. Ngo HB, Kaiser JT and Chan DC: The mitochondrial transcription and packaging factor Tfam imposes a U-turn on mitochondrial DNA. Nat Struct Mol Biol 18: 1290-1296, 2011.

4. Scarpulla RC: Transcriptional paradigms in mammalian mitochondrial biogenesis and function. Physiol Rev 88: 611-638, 2008.

5. Falkenberg M, Larsson NG and Gustafsson CM: DNA replication and transcription in mammalian mitochondria. Annu Rev Biochem 76: 679-699, 2007.

6. Campbell CT, Kolesar JE and Kaufman BA: Mitochondrial transcription factor A regulates mitochondrial transcription initiation, DNA packaging and genome copy number. Biochim Biophys Acta 1819: 921-929, 2012.

7. Gauthier BR, Wiederkehr A, Baquié M, Dai C, Powers AC, Kerr-Conte J, Pattou F, MacDonald RJ, Ferrer J and Wollheim CB: PDX1 deficiency causes mitochondrial dysfunction and defective insulin secretion through TFAM suppression. Cell Metab 10: 110-118, 2009.

8. Shi CM, Xu GF, Yang L, Fu ZY, Chen L, Fu HL, Shen YH, Zhu L, Ji CB and Guo XR: Overexpression of TFAM protects 3T3-L1 adipocytes from NYGGF4 (PID1) overexpression-induced insulin resistance and mitochondrial dysfunction. Cell Biochem Biophys 66: 489-497, 2013.

9. Kaguni LS: DNA polymerase gamma, the mitochondrial replicase. Annu Rev Biochem 73: 293-320, 2004.

10. Ruhanen H, Borrie S, Szabadkai G, Tyynismaa H, Jones AW, Kang D, Taanman JW and Yasukawa T: Mitochondrial single-stranded DNA binding protein is required for maintenance of mitochondrial DNA and 7S DNA but is not required for mitochondrial nucleoid organisation. Biochim Biophys Acta 1803: 931-939, 2010.

11. Van Tuyle GC and Pavco PA: The rat liver mitochondrial DNA-protein complex: Displaced single strands of replicative intermediates are protein coated. J Cell Biol 100: 251-257, 1985.

12. Hoke GD, Pavco PA, Ledwith BJ and Van Tuyle GC: Structural and functional studies of the rat mitochondrial single strand DNA binding protein P16. Arch Biochem Biophys 282: 116-124, 1990.

13. Korhonen JA, Gaspari M and Falkenberg M: TWINKLE Has $5 '->3$ ' DNA helicase activity and is specifically stimulated by mitochondrial single-stranded DNA-binding protein. J Biol Chem 278: 48627-48632, 2003.

14. Milenkovic D, Matic S, Kühl I, Ruzzenente B, Freyer C, Jemt E, Park CB, Falkenberg $M$ and Larsson NG: TWINKLE is an essential mitochondrial helicase required for synthesis of nascent D-loop strands and complete mtDNA replication. Hum Mol Genet 22: 1983-1993, 2013. 
15. Blomain ES and McMahon SB: Dynamic regulation of mitochondrial transcription as a mechanism of cellular adaptation. Biochim Biophys Acta 1819: 1075-1079, 2012.

16. Asin-Cayuela J and Gustafsson CM: Mitochondrial transcription and its regulation in mammalian cells. Trends Biochem Sci 32: 111-117, 2007.

17. Tauber J, Dlasková A, Santorová J, Smolková K, Alán L, Spaček T, Plecitá-Hlavatá L, Jabůrek M and Ježek P: Distribution of mitochondrial nucleoids upon mitochondrial network fragmentation and network reintegration in HEPG2 cells. Int J Biochem Cell Biol 45: 593-603, 2013.

18. Li Z, Shen J, Chen Y, Pan J, Zeng H, Fang H, Ye Z, Zeng C, Zhang R and Cai D: Mitochondrial genome sequencing of chondrocytes in osteoarthritis by human mitochondria RT2 Profiler ${ }^{\mathrm{TM}}$ PCR array. Mol Med Rep 6: 39-44, 2012.

19. Piao L, Han Y and Li D: Correlation study on adiponectin gene SNP45 and long-term oxidative stress in patients with diabetes and carotid atherosclerosis. Exp Ther Med 8: 707-712, 2014.

20. Grzybowska-Szatkowska L and Slaska B: Mitochondrial DNA and carcinogenesis (review). Mol Med Rep 6: 923-930, 2012

21. Li WH and Zheng G: Photoactivatable fluorophores and techniques for biological imaging applications. Photochem Photobiol Sci 11: 460-471, 2012

22. Hedde PN and Nienhaus GU: Super-resolution localization microscopy with photoactivatable fluorescent marker proteins. Protoplasma 251: 349-362, 2014.

23. Nienhaus GU, Nienhaus K, Hölzle A, Ivanchenko S, Renzi F, Oswald F, Wolff M, Schmitt F, Röcker C, Vallone B, et al: Photoconvertible fluorescent protein EosFP: Biophysical properties and cell biology applications. Photochem Photobiol 82: 351-358, 2006.

24. Mlodzianoski MJ, Schreiner JM, Callahan SP, Smolková K, Dlasková A, Santorová J, Ježek P and Bewersdorf J: Sample drift correction in 3D fluorescence photoactivation localization microscopy. Opt Express 19: 15009-15019, 2011.
25. Alán L, Špaček T,Zelenka J, Tauber J, Berková Z, Zacharovová K, Saudek F and Ježek P: Assessment of mitochondrial DNA as an indicator of islet quality: An example in Goto Kakizaki rats. Transplant Proc 43: 3281-3284, 2011.

26. Korr H, Thorsten Rohde H, Benders J, Dafotakis M, Grolms N and Schmitz C: Neuron loss during early adulthood following prenatal low-dose X-irradiation in the mouse brain. Int J Radiat Biol 77: 567-580, 2001.

27. Arakaki N, Nishihama T, Kohda A, Owaki H, Kuramoto Y, Abe R, Kita T, Suenaga M, Himeda T, Kuwajima M, et al: Regulation of mitochondrial morphology and cell survival by Mitogenin I and mitochondrial single-stranded DNA binding protein. Biochim Biophys Acta 1760: 1364-1372, 2006.

28. Iborra FJ, Kimura $\mathrm{H}$ and Cook PR: The functional organization of mitochondrial genomes in human cells. BMC Biol 2: 9, 2004.

29. Ban-Ishihara R, Ishihara T, Sasaki N, Mihara K and Ishihara N: Dynamics of nucleoid structure regulated by mitochondrial fission contributes to cristae reformation and release of cytochrome c. Proc Natl Acad Sci USA 110: 11863-11868, 2013.

30. Yang C, Curth U, Urbanke C and Kang C: Crystal structure of human mitochondrial single-stranded DNA binding protein at 2.4 A resolution. Nat Struct Biol 4: 153-157, 1997.

31. Antony E, Weiland E, Yuan Q, Manhart CM, Nguyen B, Kozlov AG, McHenry CS and Lohman TM: Multiple C-terminal tails within a single E. coli SSB homotetramer coordinate DNA replication and repair. J Mol Biol 425: 4802-4819, 2013.

32. Rajala N, Gerhold JM, Martinsson P, Klymov A and Spelbrink JN: Replication factors transiently associate with mtDNA at the mitochondrial inner membrane to facilitate replication. Nucleic Acids Res 42: 952-967, 2014. 